\title{
A PROOF OF KIRKMAN'S HYPOTHESIS
}

\author{
by G. N. WATSON \\ (Received 31st January 1962)
}

The theorem which I propose to establish first attracted my attention while I was turning over the pages of a volume of Cayley's Collected Mathematical Papers (Cayley, 1). The enunciation of the theorem (with no attempt towards a proof) had been published earlier by Kirkman (3) in a lengthy paper on combinatorial analysis (one of the three-score papers of which Kirkman was the author); among the topics discussed in this paper was the enumeration of the total number of different ways $D(r, k)$ in which a (convex) polygon of $r$ sides can be dissected into $k+1$ parts by drawing $k$ non-intersecting diagonals (i.e., no two diagonals may cross each other except at a vertex or outside the polygon).

The formula at which Kirkman arrived was

$$
D(r, k)=r^{k \mid 1} \times(r-k-2)^{k \mid 1},
$$

wherein the expressions such as $r^{k \mid s}$ are defined as products, thus

$$
r^{k \mid s}=r(r+s)(r+2 s) \ldots(r+k s-s) \text {. }
$$

Also he used the notation of Gamma functions in place of the corresponding factorials, his compositor representing $\Gamma$ by a peculiar curvilinear figure.

Kirkman recognised that his demonstration of this formula was incomplete and Cayley agreed with him, but neither investigated the matter further at the time. Some thirty years later, while Cayley's collected papers were in course of republication, it was possibly his paper (1) which revived his interest in the evaluation of $D(r, k)$; and in a paper (2) in which he stated that " there is no complete demonstration of this result" he published a proof which confirmed the correctness of the formula for $D(r, k)$ which Kirkman had discovered without being able to supply an adequate demonstration of it.

Kirkman's paper (3), however, contained more about $D(r, k)$ than its value which has just been quoted. He asserted that it possessed the remarkable property that

$$
D(r, k)=\frac{r}{2 k} \Sigma_{h} \Sigma_{s} D(3+h, s) D(r-h-1, k-s-1),
$$

the summations ranging from $h=0$ to $h=r-4$ and from $s=0$ to $s=k-1$; " which is the expression that I continually find. This summation I must leave to the learned and industrious reader; but, meanwhile, I shall venture to enunciate with the best demonstration, such as it is, that occurs to me the 
following Theorem" (and then he gave his statement and attempted proof of the value of $D(r, k)$ ).

Kirkman, no doubt feeling that Cayley was more competent to construct a proof of the summatory formula than he was himself, communicated his discovery to Cayley, with the consequence that Cayley published his attempt at a proof in his paper (1); thenceforth, until the construction of the proof of the summatory formula given in the present paper, nothing (so far as I know) has been written about it.

Cayley made a number of changes in the symbolism and notation used by Kirkman; but since (apart from his use of products with factors arranged in descending order, as contrasted with Kirkman's ascending factors) I consider his changes to be changes for the worse, I shall not run any risk of confusing the reader by attempting to describe them, though I shall describe his contribution to a proof of the summatory formula at the end of my paper when the reader will be better able to appreciate it.

The formula discovered by Kirkman, when written out in full, reads as follows:

$$
\begin{array}{r}
\sum_{s=0}^{k-1} \sum_{h=0}^{r-4} \frac{(3+h)^{s \mid 1}(3+h-s-2)^{s \mid 1}}{(s+1) ! s !} \cdot \frac{(r-h-1)^{k-s-1 \mid 1}(r-h-k+s-2)^{k-s-1 \mid 1}}{(k-s) !(k-s-1) !} \\
=\frac{2 k}{r} \frac{r^{k \mid 1}(r-k-2)^{k \mid 1}}{(k+1) ! k !}
\end{array}
$$

it being supposed, in view of the configuration of a polygon and sets of nonintersecting diagonals, that $r \geqq 4, k \geqq 1, k \leqq r-3$.

It is next to be observed that, for any given $s$, the product $(3+h-s-2)^{s / 1}$ contains a zero factor when $h \leqq s-1$ and that the product $(r-h-k+s-2)^{k-s-1 \mid 1}$ contains a zero factor when $h \geqq r-k+s-2$. Consequently the inner sum may be taken to run from $s$ to $r-k+s-3$ only, instead of from 0 to $r-4$.

We now make a set of changes in notation more drastic (but, $I$ think, less confusing) than those introduced by Cayley. We write

$$
k=M+1, r=M+N+4, s=m, h=m+n .
$$

With this notation the result to be established becomes

$$
\begin{aligned}
& \sum_{m=0}^{M} \sum_{m=0}^{N}\left\{\frac{(2 m+n+2) !(m+n) !}{(m+n+2) !(m+1) ! m ! n !}\right. \\
&\left.\times \frac{(2 M-2 m+N-n+2) !(M-m+N-n) !}{(M-m+N-n+2) !(M-m+1) !(M-m) !(N-n) !}\right\} \\
& \quad=\frac{2 \cdot(2 M+N+4) !(M+N+1) !}{(M+N+4) !(M+2) ! M ! N !},
\end{aligned}
$$

where $M$ and $N$ are to be regarded as any given positive integers, while $m$ 


\section{A PROOF OF KIRKMAN'S HYPOTHESIS}

and $n$ are dummy variables; Kirkman's products have been replaced by quotients of factorials.

We make further progress by introducing and studying the properties of certain generating.functions. Write.

$$
a_{m, n}=\frac{2 \cdot(2 m+n+4) !(m+n+1) !}{(m+n+4) !(m+2) ! m ! n !}, \phi(z, w)=\sum_{m=0}^{\infty} \sum_{n=0}^{\infty} a_{m, n} z^{m} w^{n},
$$

so that $a_{M, N}$ is the coefficient of $z^{M} w^{N}$ in the expansion of $\phi(z, w)$ as a double power series.

After a parenthesis in which we show that, for sufficiently small values of $|z|$ and $|w|$, this double series is absolutely convergent (so that the subsequent analysis is permissible) we proceed to express $\phi(z, w)$ as an elementary function of $z$ and $w$.

The condition which we impose on $z$ and $w$ is $|w|+2 \sqrt{ }|z|<1$, so that, a fortiori, $|w|<1$. Then the double series defining $\phi(z, w)$ is dominated by the series of positive terms

$$
\begin{aligned}
\sum_{m=0}^{\infty} \sum_{n=0}^{\infty} \frac{2 \cdot(2 m+n+4) !}{(m+2) ! m ! n !}|z|^{m}|w|^{n} \\
\quad=\sum_{m=0}^{\infty} \frac{2 \cdot(2 m+4) !}{(m+2) ! m !}|z|^{m} \sum_{n=0}^{\infty} \frac{(2 m+5)^{n \mid 1}}{n !}|w|^{n} \\
\quad=\sum_{m=0}^{\infty} \frac{2 \cdot(2 m+4) !}{(m+2) ! m !}|z|^{m}(1-|w|)^{-2 m-5} \\
=\frac{24}{(1-|w|)^{5}}\left(1-\frac{4|z|}{(1-|w|)^{2}}\right)^{-5 / 2}
\end{aligned}
$$

the first series summed being convergent when $|w|<1$, while the second series summed is convergent when

$$
4|z|<(1-|w|)^{2}
$$

and both of these conditions are satisfied in consequence of the restriction which has been imposed on $z$ and $w$.

After this digression we pass on to the simplification of $\phi(z, w)$.

The inconvenience caused by the presence of the quotient

$$
(m+n+1) ! /(m+n+4) \text { ! }
$$

in the general term of the series defining $\phi(z, w)$ is removed by the observation that

$$
\begin{aligned}
\frac{2 .(m+n+1) !}{(m+n+4) !} & =\frac{1}{m+n+2}-\frac{2}{m+n+3}+\frac{1}{m+n+4} \\
& =\int_{0}^{1} t^{m+n+1}(1-t)^{2} d t
\end{aligned}
$$


so that we may write

$$
\begin{aligned}
\phi(z, w) & =\int_{0}^{1} t(1-t)^{2} \sum_{m=0}^{\infty} \frac{(2 m+4) !}{(m+2) ! m !} z^{m} t^{m}\left(\sum_{n=0}^{\infty} \frac{(2 m+5)^{n \mid 1}}{n !} w^{n} t^{n}\right) d t \\
& =\int_{0}^{1} t(1-t)^{2} \sum_{m=0}^{\infty} \frac{(2 m+4) !}{(m+2) ! m !} z^{m} t^{m}(1-w t)^{-2 m-5} d t \\
& =\int_{0}^{1} \frac{12 t(1-t)^{2}}{(1-w t)^{5}}\left(1-\frac{4 z t}{(1-w t)^{2}}\right)^{-5 / 2} d t=\int_{0}^{1} \frac{12 t(1-t)^{2} d t}{\left\{(1-w t)^{2}-4 z t\right\}^{5 / 2}}
\end{aligned}
$$

There are perhaps half-a-dozen standard methods of evaluating an integral of this type containing a quadratic irrationality, but all of them seemed to me to require an excessive amount of calculation. The method which $I$ found to be most expeditious was to deal with the corresponding indefinite integral, writing

$$
w+2 z=\alpha, \quad 4\left(\dot{w} z+z^{2}\right)=\beta^{2}
$$

for brevity, and assume temporarily that $\alpha$ and $\beta$ are both positive. Then make the successive substitutions

and we find that

$$
t=\frac{u}{1+\alpha u}, \quad\left(u=\frac{t}{1-\alpha t}\right), \quad \beta u=\sin \theta,
$$

$$
\begin{aligned}
& \int \frac{12 t(1-t)^{2} d t}{\left\{(1-\alpha t)^{2}-\beta^{2} t^{2}\right\}^{5 / 2}}=\int \frac{12 u\{1-(1-\alpha) u\}^{2} d u}{\left(1-\beta^{2} u^{2}\right)^{5 / 2}} \\
& =\frac{12}{\beta^{4}} \int \frac{\sin \theta\{\beta-(1-\alpha) \sin \theta\}^{2} d \theta}{\cos ^{4} \theta} \\
& \quad=\frac{12}{\beta^{4}} \int\left\{\beta^{2} \frac{\sin \theta}{\cos ^{4} \theta}-2 \beta(1-\alpha) \frac{\sin ^{2} \theta}{\cos ^{4} \theta}+(1-\alpha)^{2}\left(\frac{\sin \theta}{\cos ^{4} \theta}-\frac{\sin \theta}{\cos ^{2} \theta}\right)\right\} d \theta \\
& =\frac{4}{\beta^{4}}\left[\left\{\beta^{2}+(1-\alpha)^{2}\right\} \sec ^{3} \theta-2 \beta(1-\alpha) \tan ^{3} \theta-3(1-\alpha)^{2} \sec \theta\right] .
\end{aligned}
$$

Now

$$
\begin{aligned}
\beta^{2}+ & (1-\alpha)^{2}-2 \beta(1-\alpha) \sin ^{3} \theta-3(1-\alpha)^{2} \cos ^{2} \theta \\
& =\beta^{2}-2(1-\alpha)^{2}+3 \beta^{2}(1-\alpha)^{2} u^{2}-2 \beta^{4}(1-\alpha) u^{3} \\
& =-(1-\alpha t)^{-3}\left[\left\{2(1-\alpha)^{2}-\beta^{2}\right\}(1-\alpha t)^{3}-3 \beta^{2}(1-\alpha)^{2} t^{2}(1-\alpha t)+2 \beta^{4}(1-\alpha) t^{3}\right],
\end{aligned}
$$

so that we obtain the result

$$
\begin{aligned}
& \int \frac{12 t(1-t)^{2} d t}{\left\{(1-\alpha t)^{2}-\beta^{2} t^{2}\right\}^{5 / 2}} \\
&=-4\left[\left\{2(1-\alpha)^{2}-\beta^{2}\right\}(1-\alpha t)^{3}-3 \beta^{2}(1-\alpha)^{2} t^{2}(1-\alpha t)+2 \beta^{4}(1-\alpha) t^{3}\right] \\
& \times \beta^{-4}\left\{(1-\alpha t)^{2}-\beta^{2} t^{2}\right\}^{-3 / 2}
\end{aligned}
$$




\section{A PROOF OF KIRKMAN'S HYPOTHESIS}

It follows by differentiation that this result holds whether $\alpha$ and $\beta$ are restricted to be positive or not.

When we insert the limits for $t$ we find that

$$
\begin{aligned}
\frac{\beta^{4}}{4} \int_{0}^{1} \frac{12 t(1-t)^{2} d t}{\left\{(1-\alpha t)^{2}-\beta^{2} t^{2}\right\}^{5 / 2}} & =2(1-\alpha)^{2}-\beta^{2}-2(1-\alpha) \sqrt{ }\left\{(1-\alpha)^{2}-\beta^{2}\right\} \\
& =\left[1-\alpha-\sqrt{ }\left\{(1-\alpha)^{2}-\beta^{2}\right\}\right]^{2},
\end{aligned}
$$

that is, in the original notation,

$$
4 z^{2}(z+w)^{2} \phi(z, w)=\left[1-w-2 z-\sqrt{ }\left\{(1-w)^{2}-4 z\right\}\right]^{2} .
$$

This concludes the study of $\phi(z, w)$. We now define the function $\psi(z, w)$ by the formula

$$
2 z(z+w) \psi(z, w)=1-w-2 z-\sqrt{ }\left\{(1-w)^{2}-4 z\right\},
$$

and we proceed to expand $\psi(z, w)$ as a double power series in $z$ and $w$.

Evidently we have

$$
\begin{aligned}
1-\alpha-\sqrt{ }\left\{(1-a)^{2}-\beta^{2}\right\} & \left.=-\left[\sqrt{ }\{1-\alpha)^{2}-\beta^{2} v\right\}\right]_{v}^{v}=1 \\
& =-\int_{0}^{1}\left(\frac{d}{d v} \sqrt{ }\left\{(1-\alpha)^{2}-\beta^{2} v\right\}\right) d v \\
& =\frac{\beta^{2}}{2} \int_{0}^{1} \frac{d v}{\sqrt{\left\{(1-\alpha)^{2}-\beta^{2} v\right\}}}
\end{aligned}
$$

but this last expression, though it has the advantage of exhibiting $\beta^{2}$ as a factor, suffers from the disadvantage that the integral involved is not obviously expressible as a simple double power series in $z$ and $w$.

We accordingly make a substitution, and, with the example of $\phi(z, w)$ before us, we choose the new variable $t$ so that both $v$ and $\sqrt{ }\left\{(1-\alpha)^{2}-\beta^{2} v\right\}$ are expressible as rational functions of both $t$ and $\sqrt{ }\left\{(1-\alpha t)^{2}-\beta^{2} t^{2}\right\}$.

It is a fairly natural proceeding to consider the substitution

$$
\sqrt{ }\left\{(1-\alpha)^{2}-\beta^{2} v\right\}=\frac{c_{0}+c_{1} t}{\sqrt{ }\left\{(1-\alpha t)^{2}-\beta^{2} t^{2}\right\}},
$$

where the constants $c_{0}$ and $c_{1}$ are chosen so that $v$ and $t$ vanish simultaneously, while $v=1$ when $t=1$.

This choice makes

$$
c_{0}=1-\alpha, \quad c_{0}+c_{1}=(1-\alpha)^{2}-\beta^{2},
$$

and it proves successful; in fact we find that

$$
-\frac{\frac{1}{2} \beta^{2}}{\sqrt{\left\{(1-\alpha)^{2}-\beta^{2} v\right\}}} \frac{d v}{d t}=-\frac{\beta^{2}(1-t)}{\left\{(1-\alpha t)^{2}-\beta^{2} t^{2}\right\}^{3 / 2}},
$$

whence it easily follows that

$$
1-\alpha-\sqrt{ }\left\{(1-\alpha)^{2}-\beta^{2}\right\}=\beta^{2} \int_{0}^{1} \frac{(1-t) d t}{\left\{(1-\alpha t)^{2}-\beta^{2} t^{2}\right\}^{3 / 2}} .
$$


In carrying out this substitution, it has been tacitly assumed that $\alpha$ and $\beta$ are positive with $\alpha+\beta<1$; but, now that the result has been obtained, these limitations may be discarded because we may use the formula

$$
\int_{0}^{1} \frac{d}{d t}\left(\frac{(1-\alpha)-\left(\alpha-\alpha^{2}+\beta^{2}\right) t}{\sqrt{ }\left\{(1-\alpha t)^{2}-\beta^{2} t^{2}\right\}}\right) d t=-\beta^{2} \int_{0}^{1} \frac{(1-t) d t}{\left\{(1-\alpha t)^{2}-\beta^{2} t^{2}\right\}^{3 / 2}}
$$

to obtain the desired result.

We now replace $\alpha$ and $\beta$ by their values in terms of $z$ and $w$, so that we have

$$
\begin{aligned}
\psi(z, w) & =\int_{0}^{1} \frac{2(1-t) d t}{\left\{(1-w t)^{2}-4 z t\right\}^{3 / 2}} \\
& =\int_{0}^{1} \frac{2(1-t)}{(1-w t)^{3}}\left(1-\frac{4 z t}{(1-w t)^{2}}\right)^{-3 / 2} d t \\
& =\int_{0}^{1} \frac{2(1-t)}{(1-w t)^{3}} \sum_{m=0}^{\infty} \frac{\left(\frac{3}{2}\right)^{m \mid 1}(4 z t)^{m}}{m !(1-w t)^{2 m}} d t \\
& =\int_{0}^{1}(1-t) \sum_{m=0}^{\infty} \frac{(2 m+2) !(z t)^{m}}{(m+1) ! m !} \cdot(1-w t)^{-2 m-3} d t \\
& =\int_{0}^{1}(1-t) \sum_{m=0}^{\infty} \sum_{n=0}^{\infty}\left\{\frac{(2 m+n+2) !}{(m+1) ! m ! n !}(z t)^{m}(w t)^{n}\right\} d t \\
& =\sum_{m=0}^{\infty} \sum_{n=0}^{\infty} \frac{(2 m+n+2) !(m+n) !}{(m+n+2) !(m+1) ! m ! n !} z^{m} w^{n},
\end{aligned}
$$

since

and consequently

$$
\int_{0}^{1}(1-t) t^{m+n} d t=\frac{(m+n) !}{(m+n+2) !}
$$

where

$$
\psi(z, w)=\sum_{m=0}^{\infty} \sum_{n=0}^{\infty} b_{m, n} z^{m} w^{n}
$$

We now have

$$
b_{m, n}=\frac{(2 m+n+2) !(m+n) !}{(m+n+2) !(m+1) ! m ! n !}
$$

$$
\left\{\sum_{m=0}^{\infty} \cdot \sum_{n=0}^{\infty} b_{m, n} z^{m} w^{n}\right\}^{2}=\sum_{m=0}^{\infty} \sum_{n=0}^{\infty} a_{m, n} z^{m_{i} w^{n}}
$$

identically in $z$ and $w$, since both have now been proved equal to

$$
\left\{\frac{1-w-2 z-\sqrt{ }\left\{(1-w)^{2}-4 z\right\}}{2 z(z+w)}\right\}^{2}
$$

and consequently coefficients of like powers of $z$ and $w$ on both sides of the identity are equal. Now the coefficient of $z^{M} w^{N}$ on the right is $a_{M, N}$, while the coefficient of $z^{M} w^{N}$ on the left when the series are multiplied out in accordance 
with Cauchy's rule is

so that we have

$$
\sum_{m=0}^{M} \sum_{n=0}^{N} b_{m, n} b_{M-m, N-n}
$$

$$
\sum_{m=0}^{M} \sum_{n=0}^{N} b_{m, n} b_{M-m, N-n}=a_{M, N}
$$

When we replace $a_{m, n}$ and $b_{m, n}$ by the expressions which defined them, we see that this result is precisely the result which is asserted by Kirkman's hypothesis in the notation in which I found it convenient to enunciate it.

The truth of his hypothesis is consequently established.

The only noteworthy feature of this extremely elementary proof of Kirkman's hypothesis is the amount of calculation which seems to be required in evaluating the definite integral by means of which $\phi(z, w)$ is expressed as an elementary function of $z$ and $w$.

The contribution which Cayley (1) made to the problem was a study of the special case $N=0$ (in the notation which we have used). He thus had to prove that

$$
2^{2 M+4} \sum_{m=0}^{M} \frac{\left(\frac{1}{2}\right)^{m+1 \mid 1}\left(\frac{1}{2}\right)^{M-m+1 \mid 1}}{(m+2) !(M-m+2) !}=\frac{(2 M+2) \cdot(2 M+4) !}{(M+4) !(M+2) !} .
$$

When we write $M=R-4, m=r-2$, the expression on the left becomes

$$
\begin{aligned}
& 2^{2 R-2} \sum_{r=2}^{R-2} \frac{\left(-\frac{1}{2}\right)^{r \mid 2}\left(-\frac{1}{2}\right)^{R-r \mid 1}}{r !(R-r) !} \\
& =2^{2 R-2}\left\{\sum_{r=0}^{R} \frac{\left(-\frac{1}{2}\right)^{r \mid 1}\left(-\frac{1}{2}\right)^{R-r \mid 2}}{r !(R-r) !}-2 \frac{\left(-\frac{1}{2}\right)^{R \mid 1}}{R !}-2 \frac{\left(-\frac{1}{2}\right)\left(-\frac{1}{2}\right)^{R-1 \mid 1}}{(R-1) !}\right\} \\
& =2^{2 R-2} \sum_{r=0}^{R} \frac{\left(-\frac{1}{2}\right)^{r \mid 1}\left(-\frac{1}{2}\right)^{R-r \mid 1}}{r !(R-r) !}+\frac{2^{2 R-2}\left(\frac{1}{2}\right)^{R-2 \mid 1}\left(R-\frac{3}{2}-\frac{1}{2} R\right)}{R !}
\end{aligned}
$$

To evaluate the sum, we use Vandermonde's theorem in the form

$$
\sum_{r=0}^{R}\left(\begin{array}{l}
R \\
r
\end{array}\right) x^{r \mid 1 y^{R-r \mid 1}=(x+y)^{R \mid 1}}
$$

and, when $x=y=-\frac{1}{2}$, the product on the right is evidently zero. It then follows immediately that

$$
\begin{aligned}
2^{2 M+4} \sum_{m=0}^{M} \frac{\left(\frac{1}{2}\right)^{m+1 \mid 1}\left(\frac{1}{2}\right)^{M-m+1 \mid 1}}{(m+2) !(M-m+2) !} & =\frac{(2 R-4) !(2 R-6)}{(R-2) ! R !} \\
& =\frac{(2 M+4) !(2 M+2)}{(M+4) !(M+2) !},
\end{aligned}
$$

and this is the result to be established.

Although Cayley was presumably far more familiar with Vandermonde's theorem than the majority of his contemporaries, he failed to recognise that 
the sum which he needed to evaluate was merely an example of that theorem, with the result that he replaced the sum by a hypergeometric series and then occupied about half of his paper with a ponderous proof that the sum was equal to zero. It was possibly the forbidding appearance of Cayley's work which has deterred everybody from investigating Kirkman's hypothesis in its general form for slightly more than a century.

\section{REFERENCES}

(1) A. Cayley, Phil. Mag. (4) 13 (1857), 419-423= Collected Math. Papers, 3 (1890), 250-253.

(2) A. CAYley, Proc. London Math. Soc. (1) 22 (1891), 237-262 = Collected Math. Papers, 13 (1897), 93-113.

(3) T. P. Kirkman, Phil. Trans. Royal Soc., 147 (1857), 217-272.

46 WARWICK NEW ROAD

LEAMINGTON, WARWICKSHIRE 